\title{
An Examination of Daily Information Needs and Sharing Opportunities
}

\author{
David Dearman ${ }^{1}$, Melanie Kellar ${ }^{2}$, Khai N. Truong ${ }^{1}$ \\ ${ }^{1}$ Department of Computer Science \\ University of Toronto \\ Toronto, ON M5S 3G4 Canada \\ \{dearman, khai\}@cs.toronto.edu \\ ${ }^{2}$ Google Inc. \\ 1600 Amphitheatre Parkway \\ Mountain View, CA 94043 USA \\ kellar@google.com
}

\begin{abstract}
A person often has highly context-sensitive information needs that require assistance from individuals in their social network. However, a person's social network is often not broad enough to include the right people in the right situations or circumstances who can satisfy the needs. The ability to satisfy context-sensitive information needs depends on a person's ability to seek the answers from appropriate individuals, who must then provide a response in a timely manner. To gain an understanding of how to better support the sharing of information, we conducted a four-week diary study examining 20 people's perceived daily information needs and sharing desires. We provide a structured framework for understanding the types of information people need and discuss when and how people are able to satisfy their needs. Using these findings, we discuss research and design opportunities for addressing the shortcomings of the existing information sources by connecting information altruists with an audience by leveraging weak ties through situation and circumstance, and providing a timely asynchronous connection to these sources.
\end{abstract}

\section{Author Keywords}

Diary study, information need, information sharing, design considerations, communal knowledge

\section{ACM Classification Keywords}

H5.m. Information interfaces and presentation (e.g., HCI): Miscellaneous.

\section{INTRODUCTION}

People encounter a variety of information needs during their daily lives, ranging from the mundane, (e.g., "What is the time zone in Florida?") to the complex (e.g., "A family doctor who is taking patients"). There are a variety of methods available to satisfy these information needs,

Permission to make digital or hard copies of all or part of this work for personal or classroom use is granted without fee provided that copies are not made or distributed for profit or commercial advantage and that copies bear this notice and the full citation on the first page. To copy otherwise, or republish, to post on servers or to redistribute to lists, requires prior specific permission and/or a fee.

$\mathrm{CSCW}^{\prime}$ 08, November 8-12, 2008, San Diego, California, USA.

Copyright 2008 ACM 978-1-60558-007-4/08/11...\$5.00. including the Web, offline resources (e.g., phone book) and a personal social network (e.g., ask a friend). However, people encounter information needs in their daily life such that it can be difficult to obtain an answer using current means. The information may not exist in an electronic format available online; or it may only be found through people whom one does not know or have access to [11]. For example, "[I want] to know if a stroller can be used on the Don Valley trail [given the recent snowfall]" is highly contextualized and can only be answered by someone who has recent knowledge of the trail conditions. Additionally, an individual may find it difficult to share the practical information and knowledge they accumulate through their daily experiences with an audience that will find it useful.

The study of information need traditionally has focused on the tasks users perform on the Web [5, 15, 17, 24], the goal of their search queries [3, 12-14, 22], and the use of online resources to access the communal knowledge of colleagues $[1,7,8,16,21]$. These studies typically have taken place in often frequented environments, such as the home or workplace. As a result, the types of information people need and share across all context of their daily life (e.g., while walking on the street or driving to work) is not well understood. Sohn et al. 's [25] study of mobile information needs is a notable exception.

In this paper, we report on a four-week diary study in which a diverse group of participants recorded the broad set of information they needed ${ }^{1}$ or wanted to share ${ }^{2}$ in their daily lives. Based on the diary entries, we categorize the information needs and shares into an information framework. Our results show that:

- Information needs often are situated and contextualized in nature. Participants believed that people with weak

\footnotetext{
${ }^{1}$ Information need is any information that is required for a task, or to satisfy the curiosity of the mind, regardless of whether the need is satisfied or not.

2 Information share is any information acquired throughout everyday experiences that people perceive they would like to share, regardless of whether the information is shared.
} 
ties through shared context or interests would be ideal information sources in some instances.

- If asked for the information, participants would be willing to share most of the knowledge they accumulate in everyday life. However, they currently lack the tools to do so without exerting extraordinary effort.

- The timeliness of information is a significant consideration and one reason why existing systems do not satisfy some of the participants' needs. The situated and contextualized nature of the needs means that archived answers from the past may not be applicable in some circumstances, while a delayed response that does not arrive until much later would be too late.

These findings present a new understanding of people's everyday information needs across all contexts, independent of the methods used to satisfy the needs. Informed by our results, we discuss opportunities to leverage weak ties between people to facilitate collaborative sharing of information to satisfy everyday information needs.

\section{RELATED WORK}

Previous definitions of information needs have been constructed with information retrieval (IR) systems in mind. For example, Shneiderman et al. define it as "the perceived need for information that leads to someone using an information retrieval system in the first place" [23]. Campbell states "information needs are combinations of ideas such as what the target information might look like, where it might be found, or how one might go about tracking it down" [4]. Information needs in the context of our work is when an individual requires any information to complete a task, or to satisfy the curiosity of the mind, independent of the method used to address the need, and regardless of whether the need is satisfied or not.

Recent studies of information needs commonly are grounded in IR systems and the Web. In particular, the study of Web-based information needs has focused on the tasks users perform on the Web (e.g., navigating sites, searching) $[5,15,17,24]$, and the goal of their search queries [3, 12, 13, 22]. The Web and search engines have played a key role in satisfying people's information needs, as demonstrated by the informational nature of many Web searches. Previous research has shown that informational ${ }^{3}$ searches account for anywhere from $48 \%$ [3], to 61\% [22], to $\sim 80 \%$ [13] of search queries.

The increasing availability of Web-enabled mobile devices and search services, such as Google Mobile (http:// mobile.google.com) and Yahoo! Mobile (http:// mobile.yahoo.com) can provide new opportunities for acquiring information in contexts not previously supported. Kamvar and Baluja [14] found in their analysis of Google's

\footnotetext{
${ }^{3}$ Where "The intent is to acquire some information assumed to be present on one or more web pages." [3].
}

mobile search queries that the type of information people search for on their mobile devices is different than the desktop. In an analysis of over 30 million mobile Internet requests, Church et al. [6] classified $94 \%$ of mobile Web sessions as browsing and only $6 \%$ as search requests. Sohn et al. 's [25] study of mobile information needs found that when away from home and work, people use a diverse set of methods to satisfy their information needs. Additionally, they show that $72 \%$ of information needs are influenced by current activity, location, time of the need, and conversations with other people. Whereas Sohn et al. [25] address only mobility, we are interested in gaining a better understanding of information needs in all contexts including home, work, school, and everywhere in-between.

Web and IR systems are only a subset of resources from which an individual accesses information; a person's social network is another valuable information resource. However, it is not always the case that friends, family and colleagues with whom a person has strong ties can provide assistance. As such, an individual also might seek assistance from acquaintances and strangers, with whom she has weak ties [11]. Within the workplace, computer-mediated communication has leveraged weak ties to access intellectual resources within the workplace $[1,7,8,16,21]$. Additionally, information sharing of a more public and diverse nature has grown with the advent of social networking sites such as Yahoo! Answers (http://answers.yahoo.com), Wikipedia (http://wikipedia.com), and Twitter (http://twitter.com). In addition to our focus on information needs, we explore the information that participants acquire throughout their everyday experiences that they perceive they would like to share, regardless of whether the information is shared.

\section{METHOD}

We conducted a 4-week diary study to explore the information that 20 participants needed and wanted to share in their daily lives. We chose to use diary studies because a diary offers an ecologically valid way of allowing participants to document any situation in which they would like to obtain information or provide it to others with minimal disruption to their normal activities [9]. To minimize any disturbances, we designed the diary to ask for very short and specific information and met with participants each week to elicit additional details.

\section{Participants}

Through word-of-mouth and flyers posted in the community around the University of Toronto, twenty paid participants (10 male, 10 female) were recruited. Participation was open to anyone ages 18 or older who live within Toronto, Canada. We did not enforce any other restrictions because we wanted to ensure a diverse participant sampling with respect to background and daily experiences given the broad nature of our exploration. The age of participants ranged from 18-55, with most (17) between the ages of 18 and 35. Fifteen participants were employed full-time, four were students and one was both working and studying. All participants owned a Web-enabled computer that they used daily, and a mobile 

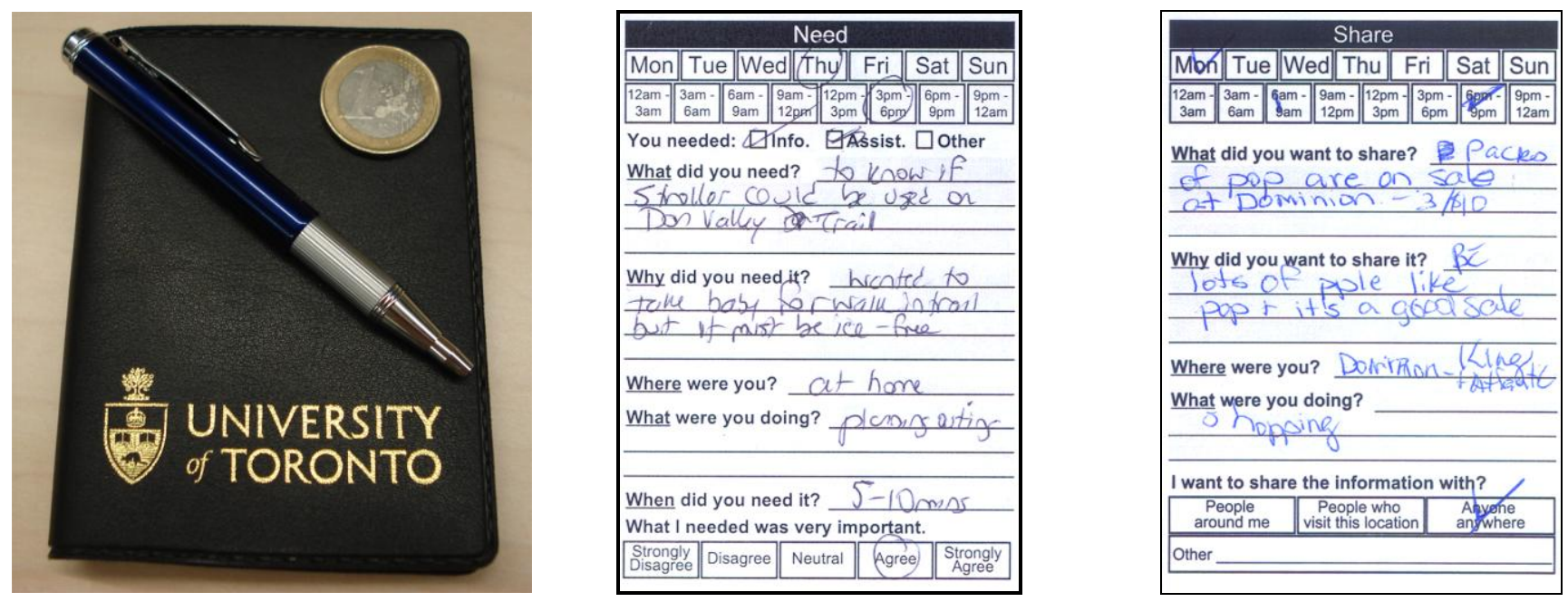

Figure 1. The diary form factor (left). An actually diary page with entry for information needs (middle) and share (right).

phone that they used daily, semi-daily or weekly. Although all participants had a Web-enabled mobile phone, they reported that they rarely (4) or never (16) use their phone to access the Web. Reasons cited included the high cost of accessing the Web on their mobile phone ${ }^{4}$, and difficulties in using their device's cumbersome interface.

\section{Procedure}

Each participant carried a small paper diary (Figure 1: left) for up to 4 weeks. We instructed participants to record into their diary all information they need for a task or to satisfy a curiosity, and any information they acquire throughout their everyday experiences that they would like to share with others. We did not constrain the scope of the information needs or sharing. Participants were free to enter any information within the diary they perceived as information they would like to have or to share. Twice a day, we sent participants a text message or email reminding them to update their diary. Only one participant chose to receive email rather than text messages. Each week, we met with participants to collect the diaries, interview them, and give them new diaries for the next week. Interviews took between 15-60 minutes and followed a semi-structured format, allowing the interviewer to probe further into the experience behind each entry. We asked each participant to explain the intent of each entry in greater detail and when applicable, the methods used to get or share the information. For share entries, we also probed with whom the participant would be willing to share the information. At the end of the final week, we conducted an exit interview with each participant to understand his or her overall experiences. The weekly and exit interviews were all audio recorded and transcribed with participant consent.

We compensated participants \$30 CAD for a minimum commitment of one week. Participants who completed the

\footnotetext{
${ }^{4}$ The monthly mobile data access rates for Canada's three major providers as of April 10, 2008: $\$ 7$ plus $\$ 0.05 / \mathrm{KB}, \$ 40$ for $8 \mathrm{MB}$ and $\$ 100$ for $1 \mathrm{~GB}$.
}

full four weeks had their names entered into a drawing for one of three $\$ 100 \mathrm{CAD}$ gift certificates.

\section{Instrument}

Despite the growing use of alternate methods to conduct a diary study (e.g., Web-forms [25], voice mailbox [18], cameras [24]) we chose to design a small pocketsize paper diary that participants carry with them. The benefit of a paper diary is that it enhances the recorder's ability to make full or partial entries in situ, rather than partial entries that they complete later, and out of context [24, 25].

Each page of the diary contains a short structured form with questions about the information the participant needed or wanted to share. The need questions (Figure 1: middle) are: day and time; What did you need?; Why did you need it?; Where were you?; What were you doing?; When did you need it?; and the importance of the need. The share questions (Figure 1: right) are: the day and time; What did you want to share?; Why did you want to share it?; Where were you?; What were you doing?; and with whom they would want to share the information. Each diary consisted of 30 need forms and 30 share forms.

A diary study does have some limitations. Self-reported information may not be complete and furthermore may contain inaccuracies. Additionally, participants may forget or choose not to report all qualifying entries. Our specific approach takes these limitations into consideration. We intentionally designed the form to be short to minimize the time burden when completing an entry. We met with participants each week to extract additional details when necessary. We also reminded participants twice a day, using text-messages or emails, to enter any experience they have yet to record. A diary study allows participants to self-filter out sensitive experiences when necessary. At the very least, the number of diary entries gathered using this technique provides a realistic lower bound for the number of times participants experienced the studied effect. 


\section{RESULTS AND DISCUSSION}

In this section, we present a unified information framework that was developed using grounded theory affinity analysis $[2,10]$ of our data. We then discuss the nuances of the information needs and shares in turn.

Eighteen of the twenty participants completed the full four weeks of the study. The remaining two participants completed three weeks. Overall, participants recorded a combined 1290 entries (858 need; 432 share), with each participant averaging 42.9 need (min: 19; max: 88; SD: 17.9) and 21.6 share (min: 5; max: 60; SD: 14.1) entries over the course of their involvement in the study. To analyze the entries, we first extracted a direct quote for each of the 1290 diary entries from the participant's handwritten notes and the interview transcripts. Two researchers then created a bottomup affinity diagram by identifying and clustering similar or related items, which was then challenged by a third researcher, followed by a round of refinement. This process repeated for three iterations until a consensus was reached.

\section{Information Framework}

We categorized all the information needs and share entries into nine primary categories: Persons, Establishments \& Organizations, Offerings, Events, Environmental Conditions, Trivia \& Pop Culture, Finding, Availability, and Guidance. Each category has at least two secondary categories, except for trivia \& pop culture. The primary and secondary categories are illustrated with examples in Table 1. The number of information needs and share entries for each category are presented in Table 2 .

Persons include information about a specific individual who is known and can be identified or oneself. We observed four distinct types of information concerning a person: their mental and physical well-being; their professional and personal background experience and

Table 1. The information categories for the need and share diary entries. The example quotes are information needs from our study.

\begin{tabular}{|c|c|c|}
\hline Information Category & Description & Example \\
\hline 1. Persons & Information about a specific person or oneself. & \\
\hline 1.1 Well-being & The mental and physical well being of a person. & "How sick is my student feeling?" \\
\hline 1.2 Background & $\begin{array}{l}\text { The professional or personal background experiences and knowledge of a } \\
\text { person. }\end{array}$ & "Is my ex-girlfriend seeing anyone?" \\
\hline 1.3 State of an asset & The current state of an asset the person owns. & "Did I forget to turn the TV off?" \\
\hline 1.4 Contact information & Their contact information. & "What is my uncles email address?" \\
\hline 2. Establishments \& Organizations & $\begin{array}{l}\text { Information about a specific business, association, society, civil department, } \\
\text { etc. }\end{array}$ & \\
\hline 2.1 Properties & $\begin{array}{l}\text { Attributes and characteristics such as staffing, finances, physical design and } \\
\text { aesthetics. }\end{array}$ & "A list of all organization members." \\
\hline 2.2 Operating procedures & Rules and regulations that govern the operating procedures. & "Does Toys-R-US allow exchanges without a receipt?" \\
\hline 2.3 Contact information & Its contact information. & "What is the phone number for EL Trompo." \\
\hline 3. Offerings & $\begin{array}{l}\text { Information about a specific product or service provided by an establishment } \\
\& \text { organization }\end{array}$ & \\
\hline 3.1 Properties & The physical design, makeup, attributes, and affordances. & "The ingredients list for a cream" \\
\hline 3.2 Pricing & The cost if applicable. & "Prices for Mount Tremblant during New Year." \\
\hline 3.3 Quality & The grade, superiority, and excellence. & "How is the ice on the skating rink at College Park?" \\
\hline 4. Events & $\begin{array}{l}\text { Information about a specific activity, function or event of importance that } \\
\text { involves people. }\end{array}$ & \\
\hline 4.1 Logistics & $\begin{array}{l}\text { The coordination, organization, management or a complex operation that } \\
\text { involves many people. }\end{array}$ & "What is the agenda for tonight's meeting?" \\
\hline 4.2 Pricing & The cost if applicable. & "How much are Edgefest tickets?" \\
\hline 4.3 Progress \& outcomes & The progression, consequences and results. & "Did Obama win more states than Clinton?" \\
\hline 5. Environmental Conditions & $\begin{array}{l}\text { Conditions of the physical environment that currently or in the future will } \\
\text { impact the inquirer. }\end{array}$ & \\
\hline 5.1 Weather & The weather conditions and future forecast. & "Is it too cold outside to go running?" \\
\hline 5.2 Traffic & Traffic conditions. & "Is there more traffic on Spadina or Harbord?" \\
\hline 6. Trivia \& Pop culture & $\begin{array}{l}\text { Seemingly random, inquisitive thoughts, factual knowledge, and pop culture } \\
\text { references that permeate our everyday life. }\end{array}$ & "What is the normal body temperature?" \\
\hline 7. Finding & $\begin{array}{l}\text { Information required to identifying and locating a person; establishment \& } \\
\text { organization; offering; event; and asset. }\end{array}$ & \\
\hline 7.1 Identify & Establish the existence of and identify by name the thing to be found. & "A family doctor who is taking patients." \\
\hline 7.2 Locate & Establish the physical presence of a specific (identified) thing. & "Where is an LCBO close to my route home?" \\
\hline 8. Availability & $\begin{array}{l}\text { Information identifying when a person is available; an organization, } \\
\text { establishment and event are accessible; and an offering or asset is obtainable. }\end{array}$ & \\
\hline 8.1 Scheduled & Availability given a defined schedule that is maintained. & "How late is Cinnabon at Union Station open?" \\
\hline 8.2 Circumstantial & $\begin{array}{l}\text { Availability that does not follow a defined schedule or resulting from factors } \\
\text { that may cause a deviation in a defined schedule }\end{array}$ & “Would Sar be at Church today?” \\
\hline 9. Guidance & The knowledge to perform an action and inform a decision. & \\
\hline 9.1 Instruction & Instructions or directions to perform a specific task & "How do you look after an Orchid?" \\
\hline 9.2 Advice \& Opinion & Advice and opinion from a knowledgeable source, to inform a task. & "Ideas for a Valentines and Anniversary gift." \\
\hline
\end{tabular}


Table 2. The total number of diary entries of both information needs and shares for each information category. Additionally, the needs and shares are separated into their respective location contexts: home, work and mobile.

\begin{tabular}{|c|c|c|c|c|c|c|c|c|c|c|c|c|c|c|c|c|}
\hline \multirow{3}{*}{ Information Categories } & \multicolumn{8}{|c|}{ Information needs } & \multicolumn{8}{|c|}{ Information shares } \\
\hline & \multicolumn{2}{|c|}{ All Entries } & \multicolumn{2}{|c|}{ Home } & \multicolumn{2}{|c|}{ Work } & \multicolumn{2}{|c|}{ Mobile } & \multicolumn{2}{|c|}{ All Entries } & \multicolumn{2}{|c|}{ Home } & \multicolumn{2}{|c|}{ Work } & \multicolumn{2}{|c|}{ Mobile } \\
\hline & \# & $\%$ & $\#$ & $\%$ & \# & $\%$ & \# & $\%$ & $\#$ & $\%$ & \# & $\%$ & \# & $\%$ & $\#$ & $\%$ \\
\hline 1. Persons & 20 & 2.3 & 11 & 3.0 & 3 & 1.4 & 6 & 2.1 & 22 & 5.1 & 11 & 6.3 & 5 & 5.6 & 6 & 2.6 \\
\hline 2. Establishments \& Organizations & 73 & 8.5 & 27 & 7.4 & 27 & 13.0 & 19 & 6.7 & 26 & 6.0 & 5 & 2.9 & 11 & 12.2 & 10 & 4.3 \\
\hline 3. Offerings & 65 & 7.6 & 24 & 6.6 & 18 & 8.7 & 23 & 8.1 & 111 & 25.7 & 31 & 17.7 & 13 & 14.4 & 67 & 29.0 \\
\hline 4. Events & 40 & 4.7 & 13 & 3.6 & 16 & 7.7 & 11 & 3.9 & 30 & 6.9 & 17 & 9.7 & 8 & 8.9 & 5 & 2.2 \\
\hline 5. Environmental Conditions & 48 & 5.6 & 33 & 9.0 & 9 & 4.3 & 6 & 2.1 & 31 & 7.2 & 7 & 4.0 & 1 & 1.1 & 23 & 10.0 \\
\hline 6. Trivia \& Pop Culture & 31 & 3.6 & 13 & 3.6 & 3 & 1.4 & 15 & 5.4 & 8 & 1.9 & 2 & 1.1 & 4 & 4.4 & 2 & 0.9 \\
\hline 7. Finding & 300 & 35.0 & 113 & 30.9 & 68 & 32.7 & 119 & 41.9 & 91 & 21.1 & 54 & 30.9 & 33 & 36.7 & 68 & 29.4 \\
\hline 8. Availability & 185 & 21.6 & 87 & 23.8 & 43 & 20.7 & 55 & 19.4 & 82 & 19.0 & 31 & 17.7 & 11 & 12.2 & 40 & 17.3 \\
\hline 9. Guidance & 96 & 11.2 & 45 & 12.3 & 21 & 10.1 & 30 & 10.6 & 31 & 7.2 & 17 & 9.7 & 4 & 4.4 & 10 & 4.3 \\
\hline Total & 858 & 100 & 366 & 100 & 208 & 100 & 284 & 100 & 432 & 100 & 175 & 100 & 90 & 100 & 231 & 100 \\
\hline
\end{tabular}

knowledge; the state of an asset they own (e.g., TV, vehicle, pet); and their contact information.

Establishments \& Organizations includes information about a specific business establishment, association, civil department, organization, etc. No distinction is made between public or private information, or if the source is external or internal. We observed three distinct types of information concerning an establishment \& organization: the properties of the entity such as staffing, financial details, physical design and aesthetics; the rules and regulations that govern the entities daily operating procedures; and contact information.

We categorize information about a specific product or service provided by establishments \& organizations as Offerings. We observed three distinct types of information about an offering: its physical and usage properties (e.g., design, ingredients, attributes, affordances); pricing (e.g., free, \$9.95, $50 \%$ off); and quality (e.g., goodness, freshness).

The Events category includes information about a specific (known) activity, function or event. Events are intrinsically based on time and occurrences. For example, events include a local intramural volleyball game, a much-anticipated U2 concert, the latest happenings in politics, etc. We observed three distinct types of information concerning an event: logistics (e.g., co-ordination, organization, management, procedures); pricing (if applicable); and progress and outcomes (e.g., result of a vote, score in a football game).

Weather and traffic is a significant consideration in the large, northern metropolitan area of our study. The study was conducted during the winter months of January and February, during which the weather did fluctuate considerably. In summer months or more temperate climates, the number of weather related information needs and shares that occur might decrease. We have defined the category Environmental Conditions to encompass traffic and weather (e.g., temperature, snow accumulation) related information. Although we do not provide a distinction between local or worldly inquiries, all entries involved environmental conditions that were currently impacting the participant or would in the future.
Trivia and Pop Culture includes sometimes seemingly random, inquisitive thoughts (e.g., "How much water will all the snow we've had amount to?'); factual knowledge (e.g., "What is a normal body temperature?"); and pop culture references (e.g., "Is the '10\% pay yourself first' rule ... on gross or take home pay?"). Information about public figures and celebrities fits within this category, not persons.

Participants reported an overwhelming need for Finding an event, establishment, offering, person or a personal asset. Finding often involves identifying and locating, which we differentiate based on the inquirer's ability to explicitly name the thing that needs to be found. For example, "Is there a store like Bruno's in East York?" and "Where is the Cup Cake Shop?" both involve trying to find an establishment. In the first example, the inquirer has yet to identify the name (or existence) of a restaurant similar to Bruno's; whereas in the second example, the name of the bakery is already know, but the location is not.

Availability includes the information required to identify when a person is available; organizations, establishments and events are accessible; and offerings or assets are obtainable. The availability of these things is defined by an established and maintained schedule or is otherwise circumstantial. For example, "What time is Costco open till?" and "What is the lineup like at the Boulevard Room?" both deal with the availability of an establishment. However, the operating hours of Costco is pre-defined, whereas the lineup at Boulevard Room is circumstantial, changing constantly. The types of scheduled information we observed are operating hours, appointments, etc. Availability based on circumstance involves the intentions and plans of a person; the ability to obtain and use an asset; operating delays and the closure of establishments because of weather and holidays.

Guidance includes the instruction required to perform a specific task (e.g., assembly, directions, care), and the advice \& opinion from a knowledgeable source concerning a future action (e.g., gift idea, product review). Advice \& opinion permeates entries observed in all other categories. Thus, advice \& opinion is not a mutually exclusive category. We classified an entry into a category other than advice $\&$ opinion when the participant expressed (in the 
interview) that the need for or expression of an opinion was not their primary intent.

\section{Purpose of the Information Needs}

Independent of location ${ }^{5}$, the interviews and the context of the inquiries revealed that the purpose of the information needs was of a personal $(87.3 \%, 749 / 858)$ or work/school $(12.7 \%$, $109 / 858)$ nature. The majority of the personal $(82.1 \%$, $615 / 749)$ and all the work/school (100\%, 109/109) information needs were to support a task, such as: making a decision; performing an action; compiling information; or to find a thing, asset or location. Work and school tasks differed from personal tasks in that work/school tasks were not typically of the participants own choosing; rather these tasks were assigned to them by someone else, or required to complete their job activities. However, we did observe that $4.3 \%$ (32/749) of the participants personal information needs were not for themselves, but for a friend or family member. In such cases, the participants were acting as a proxy, retrieving information and then relaying it to the requester. Although the majority of information needs were motivated by a task or on the bequest of someone else, many $(13.6 \% ; 102 / 749)$ were simply to acquire knowledge or satisfy a curiosity.

\section{Timeliness of Information Needs}

The timeliness of information needs is an important consideration when choosing to address them [25]. Although some information needs are required immediately to support the task at hand, other needs do not require the same level of immediacy and therefore can be satisfied at a later time, using the most convenient method available. For each entry in the diary, we asked the participants to identify when they required the information. How to record when the information is needed was left open-ended, allowing the participants to define 'when' as they perceived it.

\section{Deadline for the Information}

The timeframe within which participants required or preferred to have their information needs satisfied varied from "immediately", "1 hour", "tonight", "next week", to "anytime". Rather than presenting each individual timeframe, we have grouped the needs into seven timeframes as illustrated in Table 3.

Information that is needed "immediately" or as soon as possible ( "ASAP") represents $42.3 \%$ (354/858) of the need diary entries. These needs typically resulted from a task that was presently of importance:

"Is there a Shoppers Drug Mart on St. George Street? I was about to leave and I was already running late ... I wanted it right away."

\footnotetext{
5 We categorized three location contexts: entries that occurred within the participant's home; entries that occurred within their work place; and mobile entries that occurred everywhere in between. Although, our participant sampling did include some students, we do not provide a distinct category for school. Rather, we combined school and work because both are secondary locations where participants regularly visit and spend a significant amount of time.
}

Table 3. Timeliness of the information needs as defined by the participants.

\begin{tabular}{l|r|r|r|r|r|r|r|r|}
\cline { 2 - 9 } & \multicolumn{2}{c|}{ All Entries } & \multicolumn{2}{c|}{ Home } & \multicolumn{2}{c|}{ Work } & \multicolumn{2}{c|}{ Mobile } \\
\cline { 2 - 9 } & \multicolumn{1}{c|}{$\#$} & \multicolumn{1}{c|}{$\%$} & \multicolumn{1}{c|}{$\#$} & \multicolumn{1}{c|}{$\%$} & \multicolumn{1}{c|}{$\%$} & \multicolumn{1}{c|}{ \% } & \multicolumn{1}{c|}{$\%$} \\
\hline Immediate & 194 & 22.6 & 54 & 14.8 & 40 & 19.2 & 100 & 35.2 \\
ASAP & 160 & 18.6 & 65 & 17.8 & 49 & 23.6 & 46 & 16.2 \\
2-3 hours & 178 & 20.7 & 80 & 21.9 & 37 & 17.8 & 61 & 21.5 \\
Today & 73 & 8.5 & 28 & 7.7 & 25 & 12.0 & 20 & 7.0 \\
2-3 days & 107 & 12.5 & 56 & 15.3 & 26 & 12.5 & 25 & 8.8 \\
Beyond & 65 & 7.6 & 39 & 10.7 & 12 & 5.8 & 14 & 4.9 \\
Anytime & 81 & 9.4 & 44 & 12.0 & 19 & 9.1 & 18 & 6.3 \\
\hline \multicolumn{1}{r}{ Total } & 858 & 100 & 366 & 100 & 208 & 100 & 284 & 100 \\
\hline
\end{tabular}

Although immediacy does not represent the majority of the information needs, it was the highest single reported timeframe. The majority of information needs did not have a similar level of immediacy $(58.7 \%, 504 / 858)$; rather, they could be addressed at a later, convenient time:

"I want to know the price of hair styling ... to determine whether or not I could afford the style I want. I thought about it in the morning, but didn't look it up till later during a break."

A small percentage $(9.4 \%)$ of the information needs did not have any expressed timeframe imposed upon them by the participants. Rather these needs could be satisfied "anytime" or "whenever". Typically, these needs were driven by random curiosity, rather than supporting a task:

\section{"Who makes the Obay ads? ... I was curious."}

The timeliness of information needs is not independent of the location of inquiry. Immediate information needs represent $35.2 \%$ of mobile diary entries, compared to $19.2 \%$ at work, and $14.8 \%$ at home (see Table 3 ). Similarly, $38.0 \%$ of information needs within the home have a timeliness of two to three days and beyond, compared to $27.4 \%$ at work, and $20.1 \%$ when mobile. Overall, these results highlight an important difference in the timeliness of information across home, work and mobility. Additionally, the greater immediacy of mobile information needs suggests that the needs are more relevant to the task at hand, therefore requiring answers more quickly.

Although some information needs were required by a firm deadline, others were more flexible. For example, one participant wanted to find a deli that served a sandwich she liked, but she could not find one in time for lunch that day. Although she did not satisfy the information need in time for lunch, she reported that the information was still useful for the future:

"I was thinking about it for lunch, but I wanted it in general, not just that day. I would still want to know if someone could tell me a day later."

Timeliness as an Event

Rather than quantify the timeliness of the need in terms of minutes, hours, days, or weeks, some participants defined need deadlines by the occurrence of an event: "before I leave", "[the] next game", "before the snow [starts]", 
Table 4. How participants satisfied their information needs in time, and eventually.

\begin{tabular}{|c|c|c|c|c|c|c|c|c|c|}
\hline & \multicolumn{2}{|c|}{ All Entries } & \multicolumn{2}{|c|}{ Home } & \multicolumn{2}{|c|}{ Work } & \multicolumn{2}{|c|}{ Mobile } \\
\hline & & \# & $\%$ & $\#$ & $\%$ & \# & $\%$ & $\#$ & $\%$ \\
\hline \multirow{4}{*}{ 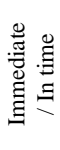 } & Ask & 217 & 51.3 & 79 & 40.5 & 68 & 56.7 & 70 & 64.8 \\
\hline & Go there & 21 & 5.0 & 3 & 1.5 & 5 & 4.2 & 13 & 12.0 \\
\hline & Web & 158 & 37.4 & 96 & 49.2 & 43 & 35.8 & 19 & 17.6 \\
\hline & Other & 27 & 6.4 & 17 & 8.7 & 4 & 3.3 & 6 & 5.6 \\
\hline \multirow{4}{*}{ 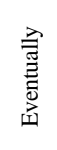 } & Ask & 49 & 23.7 & 13 & 20.6 & 19 & 38.8 & 17 & 17.9 \\
\hline & Go there & 94 & 45.4 & 24 & 38.1 & 16 & 32.7 & 54 & 56.8 \\
\hline & Web & 30 & 14.5 & 13 & 20.6 & 8 & 16.3 & 9 & 9.5 \\
\hline & Other & 34 & 16.4 & 13 & 20.6 & 6 & 12.2 & 15 & 15.8 \\
\hline
\end{tabular}

“While I am there.". Although only a small percentage of the entries $(2.3 \%, 20 / 858)$ were defined this way, it is evident from the interviews and the needs themselves that many more could have also been defined by an event. For example, a participant wanted to know if "MEC has the stainless steel water bottles in yet" while she was walking to MEC. The participant indicated that she wanted the information " $A S A P$," but the timeliness of the need could also have been specified as before she arrives at MEC.

\section{When and How the Needs Were Satisfied in Time}

Participants were able to satisfy $45.3 \%$ (389/858) of their information needs (see Table 5 and 5) either immediately $(26.8 \%)$ or in time $(19.7 \%)$. When at home $(51.4 \%)$ and work $(52.0 \%)$, participants reported greater success than when mobile (36.3\%). The participants' lack of success in satisfying their mobile needs highlights the difficulties of accessing information in a mobile context. None of the participants used the Internet on their mobile device, but all of them commented that having mobile Internet access would have been beneficial. However, the participants in Sohn et al. 's diary study found that mobile Internet access was not adequate for their information needs [25].

Participants were able to satisfy their information needs in a number of ways (see Table 5): they would ask someone (e.g., phone, in person, email, online messaging, text message); go there, to the location; look on the Web; and use other methods such as GPS, paper documents, trial and error, and print/radio/TV media. Asking another person with whom the participant has weak or strong ties was used to satisfy the majority $(51.3 \%)$ of needs, followed by the Web $(37.4 \%)$. Forty-eight of the information needs (34 immediately/in time; 14 eventually) were satisfied using multiple sources.

The location of information needs appears to have an influence on the methods used to satisfy the need (see Table 4). Participants used the Web to satisfy more information needs when at home $(49.2 \%)$ than at work $(35.8 \%)$ or while mobile (17.6\%). However, when mobile, participants

Table 5. When the information needs were satisfied.

\begin{tabular}{|c|c|c|c|c|c|c|c|c|}
\hline & \multicolumn{2}{|c|}{ Entries } & \multicolumn{2}{|c|}{ Home } & \multicolumn{2}{|c|}{ Work } & \multicolumn{2}{|c|}{ Mobile } \\
\hline & $\#$ & $\%$ & $\#$ & $\%$ & $\#$ & $\%$ & $\#$ & $\%$ \\
\hline Immediately & 230 & 26.8 & 121 & 33.1 & 59 & 28.4 & 50 & 17.6 \\
\hline In time & 159 & 18.5 & 67 & 18.3 & 49 & 23.6 & 53 & 18.7 \\
\hline Not in time & 193 & 22.5 & 60 & 16.4 & 43 & 20.7 & 90 & 31.7 \\
\hline Never & 276 & 32.2 & 118 & 32.2 & 57 & 27.4 & 91 & 32.0 \\
\hline Total & 858 & 100 & 366 & 100 & 208 & 100 & 284 & 100 \\
\hline
\end{tabular}

typically relied on other people $(64.8 \%)$ and their ability to go to a location $(12.0 \%)$.

\section{Why a Need Was Not Satisfied}

Despite the perceived importance of the reported information needs $(\mu=3.87, \sigma=0.85$, where $1=$ not important at all \& $5=$ very important), the majority of participants' reported information needs were not satisfied by the time they wanted (Not in time: $22.5 \%, 193 / 858$ ), or never satisfied (Never: 32.2\%, 276/858). The reasons for not satisfying the needs are as unique as the needs themselves. For some participants, they believed that the information could be found (online or elsewhere), but required too much effort:

Need: "A free or open source software that does resourcing that is not Microsoft Project."

Comment: "I didn't want to take the time or energy to look. Trying to find all the ones and then figure out if they are any good. I just wanted someone to tell me that they had reviewed them all, or 800 people have reviewed them all and this is what they liked the best."

Others tried searching the Web, but struggled to define their need in terms that they could use to search online:

Need: "Are there any sub $\$ 100$ snow pants in downtown Toronto?"

Comment: "I found them in the back corner of Sears. I just happened to come across them. I was so baffled I couldn't find them online."

Some believed that by taking the time to satisfy their need, they would miss the opportunity the need would satisfy:

Need: "What time does Second Cup close?"

Comment: "It was open till at least 10. I was worried it was going to close at 10, and I only had 5 minutes. If I took the time to look it up then I would have missed it."

Even with a knowledgeable source available, some participants still took a chance, perceiving their need as insignificant enough to warrant bothering a proxy for help:

Need: “Where can I get 'The Count of Monte Cristo' on DVD the cheapest."

Comment: "It was a chance thing. We went to a place and found it. We checked all the places near me but I couldn't find it. I wanted to find a place close to where I live ..."

The weather and its impact on the environment are heavily reported online and in the media; however, the finergrained weather details that are not reported may have a greater impact on people's lives:

Need: "To know if a stroller could be used on the Don Valley Trail."

Comment: "We were going to go there for a walk. Some days the trail is fine, but some days there are blocks of ice 
and I can't push the stroller over that. And there is no way to find out [ahead of time]."

For others, they believed the information would not be available online because it is highly circumstantial. Additionally, they could not identify a knowledgeable source to provide them with the information:

Need: "Is it too cold to go running?"

Comment: "I turned on the TV, but that doesn't really help runners. The best way to get it would be from another runner. Someone out running, or just back from a run."

\section{Opinion and Personal Feedback}

As previously mentioned, advice \& opinion permeated the information needs $(10.6 \%, 91 / 858)$ and sharing $(20.8 \%$, 90/432) in all categories, with the greatest concentrations in offerings (need: 6; share: 59), finding (need: 45; share: 5), and guidance (need: 30; share: 16). Participants were able to satisfy $37.4 \%(34 / 91)$ of their information needs that required an opinion. When participants did want an opinion, most commented they did not want just any opinion, but the opinion of "someone you know and trust". Although the opinions participants got from people within their social network were trustworthy, the opinions they accessed online were not as "... trust worthy [as] from someone we know".

Opinions are not set in stone; they can change over time given new experiences. One of the participants, soon after going to a restaurant expressed her satisfaction with the food ("Happy Panda has really good quality and selection"), but changed her opinion later in the day ( "Happy Panda isn't that good after all. I wanted to retract my previous statement.").

With Whom Did Participants Actually Share Information? Participants recorded 432 diary entries of information they perceived they would like to share with others. In the weekly interview, participants revealed that they did share $74.8 \%$ (323/432) of their entries; 73 with more than one group or persons. The groups and persons include: friends (40.4\%, 160/396); family $(21.2 \%, 84 / 396)$ a significant other $(11.6 \%, 46 / 396)$; work acquaintances (17.4\%, $69 / 396)$; acquaintances within their social circle $(6.6 \%$, 26/396); random strangers they came into contact with throughout the day $(1.3 \%, 5 / 396)$; and unknowns online using either Twitter or an online form $(1.5 \%, 6 / 396)$.

With Whom Are Participants Willing to Share Information? In the weekly interview, we asked participants if they would be willing to share the information they entered in the diary with a broader audience. The majority of entries $(73.6 \%, 318 / 432)$ participants indicated that, “... if capable, I would share this [information] with anyone". However, for approximately $1 / 3$ of these entries (107/318), participants clarified by adding, “... if it is relevant to them", citing location (61), activity (27), group (12), ownership of a thing (4), and interests (3) as constraints. For all other remaining entries (26.4\%; 114/432), participants only wanted to share with a specific person or closed group of people:

\section{"I just want to keep this with friends."}

Despite lacking the methods to share the information, all of the participants (20/20) stated that, "If someone would have asked me, I would have told them". If asked for the information they would be willing to share it with anyone.

However, only $1 \%$ of overall share entries included a population that can be defined as anyone. Overwhelmingly participants indicated that "the effort to share seemed like too much"; "I wasn't aware of an appropriate audience”; "I haven't run into someone who would be interested"; or "I don't have a way to do this. I don't know how you could [share the information]". Participants wanted to share the information, but they lacked the means to identify an audience and the tools to share it with them.

\section{The Implicit Sharing of Multiple Pieces of Information}

As shown in Table 2, the percentage of share entries distributed across the different information categories is relatively consistent $(+/-4 \%)$ with the number of need entries, except offerings $(+18.1 \%)$ and finding $(-13.2 \%)$. A close examination of the 111 diary entries in the offerings category reveals that many of the share entries implicitly disclose additional information relevant across categories. Specifically, 55 of the offering entries pertain to quality, of which $51(92.7 \% ; 51 / 55)$ involve an opinion. Additionally, $22(40.0 \% ; 22 / 55)$ of the quality entries involve implicitly identifying and/or locating an event, establishment or offering, satisfying possible information needs that can be categorized within finding. For example:

\section{"Manic Coffee on Bathurst and College has the best drip} coffee!! And yummy espresso too."

In this example, the participant's intent is to express their approval of the quality of Manic's drip coffee and espresso. However, the participant also discloses the location of Manic Coffee. In general, multiple pieces of information can be gleaned from how a person exactly expresses the information they wish to share.

\section{RESEARCH AND DESIGN OPPORTUNITIES}

The results above suggest three research and design opportunities: the importance of considering how to provide timely asynchronous connections to information sources and how to connect information altruists with an audience by leveraging weak ties through situations and circumstances.

\section{Weak Ties through Situations and Circumstances}

Many daily information needs are contextualized; dependent on the environment and the people in it; influenced by the activities we perform; and constrained by time. For example, "Is it too cold to go running today?", "Is there more traffic on Spadina or Harbord?", and "... if there is a line-up at Grace O'Malley's?" If one knows someone who is in the situation or location who can help, these questions are trivial to answer. Most of the time, however, they are extremely challenging. When social 
networks cannot provide the necessary information, people could leverage the diverse experiences, situations and locations of strangers. Arguably, someone may be in an appropriate situation to satisfy a person's information needs: for example, a runner who has just returned from a run; a pedestrian in the area of Spadina or Harbord, or a motorist in traffic; or a patron of Grace O'Malley's who is waiting in line, or in the bar. Search and expertise systems can be extended to identify and connect situation experts (people in the right place at the right time) with relevant information seekers. However, the challenge lies in how to identify these people. One approach may be through the use of context sensing, which could identify people in the appropriate context or with the right knowledge to support other individuals' current needs.

\section{Timely Asynchronous Connections to Information Sources}

The timeliness of information is a significant consideration and one reason why existing systems do not always adequately support time-dependent information needs. For example, when people want information about the impact of the weather on a walking trail, they want to know right away. An archived answer from last week is not relevant, and a response that arrives days or even hours later would be too late.

Providing users with the ability to define the timeliness of their information need can improve the relevance of the need and save both askers and answers time. Similarly, information with time relevance must also be stored as such. Search engines, and question and answer communities, which already act as portals for information needs, can be augmented to include situation searching and provide a better understanding of the context surrounding the information and the need. For example, the line-up at Grace O'Malley's will change with each new person who arrives or is permitted entrance. These mediums will need to identify when the information has become stale, requesting a persons help to refresh the knowledge.

It is also important to consider that an opinion is not set in stone; it can change over time and with new experiences. Systems that allow users to express their opinions should take time and new related experiences into consideration. Rather than recording an opinion as is, it can be beneficial to offer users the ability to augment their opinions at a later time.

\section{Connecting Information Altruists with an Audience}

The share diary entries reveal that participants have access to a great deal of everyday information. They perceive this information to be useful to others, yet they do not share it. Participants indicated that they would be willing to share $74 \%$ of their share diary entries with anyone, but they lack the tools and motivation to do so. Communities built to support everyday information needs, and the systems designed to connect the individuals must exploit people's willingness to share information, but do so in such a way that the cost of sharing information is minimal.

Current question and answer communities operate on the assumption that members will seek out a question to answer. However, when people are engaging in everyday activities (e.g., shopping, walking down the street), their attention will undoubtedly be focused on the tasks at hand, not if they can answer a question for someone. Rather than placing the burden on individual users to find the questions they can satisfy, novel information systems could identify the appropriate situation experts, and explicitly pose the information need to them. All of the participants (20/20) indicated that they would be willing to share their knowledge with someone (even a stranger) if asked for it. However, the act of answering a question is not without cost. Prior research has explored the motivation to contribute to online communities $[19,20]$, however, the motivation of an individual to answer questions such as “... is there a line-up at Grace O'Malley's?" will arguably be different; particularly when $42.3 \%$ of the information needs required "immediate" responses.

\section{CONCLUSION}

People frequently encounter situations in which they need information from or would like to share information with other people. Some information needs can be answered using online and offline resources or social networks. However, other situations remain that may be difficult to satisfy through current means because the needs are situated and contextualized, requiring the help of others in the right situation or circumstances. Inversely, people may help if asked, but lack the tools and general motivation to provide information to a larger audience. To gain a better understanding of the broad spectrum of people's everyday information needs, and the information they would like to share, we conducted a 4-week diary study with 20 participants. Using the 1290 unique diary entries participants record, we developed a nine-element information framework. The framework is a tool that can be leveraged in the design of future collaborative information systems. The majority of participant's information needs and sharing involved finding, availability and guidance; sharing was also heavily focused on offerings. These categories provide insight into the type of information people would like to have and to share the most, presenting opportunities for future research to explore in detail what people $d o$ require and share.

These findings present a new understanding of individual and collaborative information needs independent of the methods used to satisfy the needs. In practice, many of the participants' everyday information needs could not be satisfied because they are too situated and contextualized in nature. For those instances, participants perceived that people with whom they have weak ties through shared context or interests would be ideal information sources to satisfy their information needs. If asked for the information, participants would be willing to share most of the (non-personal) knowledge they accumulate throughout their daily lives. The timeliness of information is a significant consideration and one reason why existing systems cannot address all information needs. Again, the situated and contextualized 
nature of the needs means that archived answers from the past may not be applicable in some circumstances while a delayed response that arrives much later would be too late. Informed by our results, we described opportunities to leverage situation experts and people with weak ties to facilitate collaborative sharing of information to satisfy everyday information needs.

\section{ACKNOWLEDGEMENTS}

We thank Elaine Huang, Gillian Hayes and Julie Kientz for their feedback on this paper. This research is supported in part by NSERC. It is covered under the Ethics Review Office's protocol reference \#21610 at the University of Toronto.

\section{REFERENCES}

1. Ackerman, M.S. Augmenting the organizational memory: a field study of answer garden. In Proc. CSCW 1994, ACM Press (1994), 243-252.

2. Beyer, H., Holtzblatt, K. Contextual Design: Defining Customer-Centered Systems. Morgan Kaufmann, 1992.

3. Broder, A. A taxonomy of web search. SIGIR Forum, 36, 2 (2002), 3-10.

4. Campbell, I. The ostensive model of developing information needs. PhD thesis, University of Glasgow, September 2000.

5. Choo, C.W., Detlor, B. Turnbull, D. Information seeking on the Web: an integrated model of browsing and searching. First Monday 5, 2 (2000). Accessed April 10, 2008 from http://www.firstmonday.org/issues/issue5_2/choo

6. Church, K., Smyth, B., Cotter, P., Bradley, K. Mobile information access: a study of emerging search behavior on the mobile Internet. ACM Trans. Web 1, 1 (2007), 4.

7. Constant, D., Kiesler, S., Sproull, L. What's mine is ours, or is it? A study of attitudes about information sharing. Information Systems Research 5, 4 (1994), 400-421.

8. Constant, D., Sproull, L, Kiesler, S. The kindness of strangers: the usefulness of electronic weak ties for technical advice. Organization Science 7, 2 (1996), 119-135.

9. Czerwinski, M., Horvitz, E., Wilhite, S. A diary study of task switching and interruptions. In Proc. CHI 2004, ACM Press (2004), 175-182.

10. Glaser, B.G., Basics of grounded theory analysis: emergence vs. forcing. Sociology Press (1992).

11. Granovetter, M. The strength of weak ties. American Journal of Sociology 78, 6 (1973), 1360-1380.

12. Jansen, B.J., Spink, A., Pedersen, J. A temporal comparison of AltaVista web searching. Journal of the
American Society for Information Science and Technology 56, 6 (2005), 559-570.

13. Jansen, B.J., Booth, D.L., Spink, A. Determining the informational, navigational and transactional intent of Web queries. Information Process and Management 44, 3 (2008), 1251-1266.

14. Kamvar, M., Baluja, S. A large scale study of wireless search behavior: Google mobile search. In Proc. CHI 2006, ACM Press (2006), 701-709.

15. Kellar, M., Watters, C., Sheppard, M. A field study characterizing web-based information-seeking tasks. Journal of the American Society for Information Science and Technology 58, 7 (2007), 999-1018.

16. McDonald, D.W., Ackerman, M.S. Just talk to me: a field study of expertise location. In Proc. CSCW 1998, ACM Press (1998), 315-324.

17. Morrison, J.B., Pirolli, P., Card, S.K. A taxonomic analysis of what world wide web activities significantly impact people's decisions and actions. Ext. Abstracts CHI 2001, ACM Press (2001), 163-164.

18. Palen, L., Salzman, M. Voice-mail diary studies for naturalistic data capture under mobile conditions. In Proc. CSCW 2002, ACM Press (2002), 87-95.

19. Raban, D., Rafaeli, S. Investigating ownership and the willingness to share information online. Computers in Human Behavior 23, 5 (2007), 2367-2382.

20. Rafaeli, S., Raban, D. Information sharing online: a research challenge. International Journal of Knowledge and Learning 1, 1/2 (2005), 62-79.

21. Ribak, A., Jacovi, M., Soroka, V. "Ask before you search": peer support and community building with ReachOut. In Proc. CSCW 2002, ACM Press (2002), 126-135.

22. Rose, D., Levinson, D. Understanding user goals in web search. In Proc. WWW 2004, ACM Press (2004), 13-19.

23. Shneiderman, B., Byrd, D., Croft, W.B. Clarifying search: a user-interface framework for text searches. DLib Magazine (1997). Accessed April 10, 2008 from http://dlib.org/dlib/january97/retrieval/01shneiderman.html

24. Sellen, A.J., Murphy, R., Shaw, K.L. How knowledge workers use the web. In Proc. CHI 2002, ACM Press (2002), 227-234.

25. Sohn, T., Li, K.A., Griswold, W.G., Hollan, J. A diary study of mobile information needs. In Proc. CHI 2008, ACM Press (2008), 433-442. 